\title{
Acuerdo de mejoramiento institucional Plan de mejoramiento Fortalece la educación a distancia
}

\author{
Rectoría, Universidad Estatal a Distancia
}

\author{
Por una Universidad con mejor acceso, \\ cobertura y equidad real para nuestra población estudiantil. \\ Luis Guillermo Carpio Malavasi, Rector
}

Recibido: 1 de Febrero 2013 • Aceptado: 12 de Abril 2013

La Universidad Estatal a Distancia (UNED) está inmersa en un contexto nacional e internacional con nuevos retos y oportunidades. Consciente de los nuevos desafíos, de su función social y su liderazgo en la educación a distancia; la UNED y el gobierno de Costa Rica suscribieron el Acuerdo de Mejoramiento Institucional (AMI) para la ejecución del Plan de Mejoramiento Institucional (PMI). Este plan es el resultado de un proceso abierto de consulta en el que participó la comunidad universitaria y que tuvo su origen en el marco de las negociaciones para el Fondo Especial para el Financiamiento de la Educación Superior Estatal (FEES), donde participaron las cuatro universidades públicas adscritas al Consejo Nacional de Rectores (CONARE), y en donde adicionalmente se acordó fortalecer los procesos de inversión de las universidades en áreas estratégicas con una operación de crédito por $\$ 200$ millones, distribuidos en partes iguales entre las cuatro universidades.

Con una clara convicción en defensa de la autonomía universitaria, el AMI tiene como propósito especificar los compromisos que la UNED ha adquirido para la ejecución del financiamiento obtenido por el gobierno de Costa Rica por el monto de \$50 millones. El PMI, parte integral del AMI, contempla nueve iniciativas con las que se fortalece la presencia de la institución en las regiones alejadas del Valle Central, abriendo nuevas oportunidades para garantizar el acceso, la cobertura y la equidad a una formación universitaria de calidad para nuestra población estudiantil. Las estrategias de inversión, tendrán un impacto directo en la creación de una red de Centros Universitarios para la innovación, el desarrollo local y nacional, en el desarrollo de recursos didácticos digitales y el aprovechamiento de las tecnologías de la información y comunicación, así como en la diversificación de la oferta académica y la formación y la capacitación en el modelo de educación a distancia.

\section{Plan de Mejoramiento Institucional}

El PMI se plantea como una respuesta a las necesidades en docencia, producción, investigación y extensión. Las acciones se enmarcan dentro de la modalidad de educación a distancia y de acuerdo a los objetivos nacionales que se asignan al sistema de las universidades públicas; definidos a partir de las políticas académicas establecidas en el Plan Nacional de la Educación Superior Universitaria Estatal (PLANES) y de 
Distribución de Centros Universitarios Universidad Estatal a Distancia de Costa Rica

$w \sum_{S}^{N}$

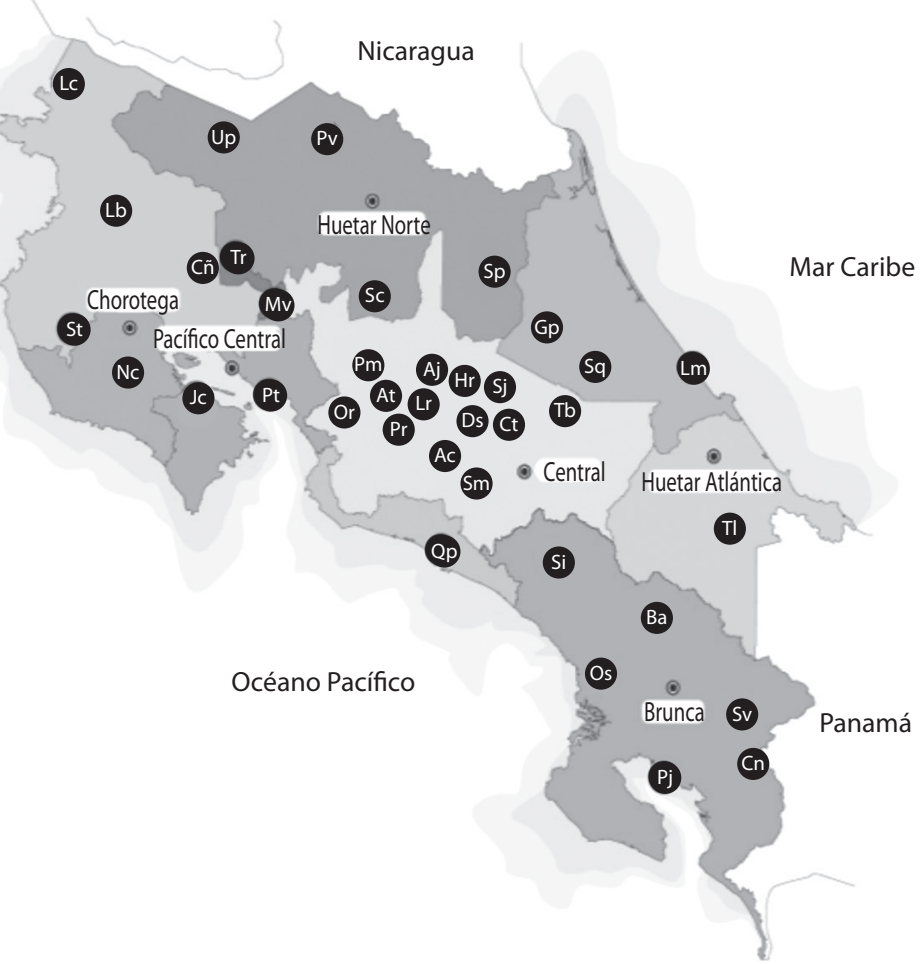

TÂ Centroos Unhiversitáaios

Ac: Acosta Os: Osa

Aj: Alajuela $\quad$ Pj: Puerto Jiménez

At: Atenas Pm: Palmares

Ba: Buenos Aires Pr: Puriscal

Cn: Ciudad Neily Pt: Puntarenas

Cñ: Cañas Pv: Pavón

Ct: Cartago Qp: Quepos

Ds: Desamparados Sc: San Carlos

Gp: Guápiles Si: San Isidro

Hr: Heredia Sj: San José

Jc: Jicaral Sm: San Marcos

Lb: Liberia Sp: Sarapiquí

Lc: La Cruz Sq: Siquirres

Lm: Limón St: Santa Cruz

Lr: La Reforma Sv: San Vito

Mv: Monteverde Tb:Turrialba

Nc: Nicoya Tl: Talamanca

Or: Orotina Tr:Tilarán

Up: Upala

las políticas públicas determinadas tanto por el Plan Nacional de Desarrollo (PND); así como por el reciente Plan Nacional de Ciencia, Tecnología e Innovación.

La UNED, en su Plan de Desarrollo Institucional 2011-2015, apuesta a una estrategia de fortalecimiento inteligente y sostenido de las Tecnologías de Información y Comunicación (TIC) en su modelo educativo; que permita modernizar tecnológicamente los procesos administrativos y académicos y responder a las necesidades y requerimientos de la comunidad universitaria y del país, de manera oportuna y pertinente, de acuerdo a los sistemas más modernos de enlace y acceso a redes. La universidad, consciente de la importancia del uso masivo de las TIC y del funcionamiento generalizado de las redes sociales, para el mejoramiento permanente en la calidad de sus carreras y de su gestión interna; orienta sus recursos a fortalecer aspectos tales como:

1. Desarrollo de propuestas innovadoras en áreas tecnológicas o metodológicas que impacten su oferta académica, fortalezcan el modelo a distancia institucional y las técnicas de aprendizaje (nuevas plataformas virtuales, dispositivos móviles, participación de redes sociales y digitalización de su material didáctico, entre otros).

2. Fortalecimiento e impulso a la capacidad tecnológica y de conectividad en los Centros Universitarios, adecuándose y aprovechando las plataformas existentes de banda ancha, teóricamente ya accesibles, pero no 
aprovechadas aún en su totalidad por debilidades tecnológicas. Eso permitiría ofrecer una prestación más oportuna y eficiente de servicios universitarios que promuevan la cobertura y la equidad.

3. Fortalecimiento de la labor universitaria de los Centros Universitarios como gestores de cambio, apostando inicialmente a su desarrollo tecnológico e infraestructura, pero con una mirada puesta en su función social como dinamizadores locales de desarrollo y propiciadores de nuevas formas de socialización regional en redes.

4. Desarrollo tecnológico integral de la institución y también de los sistemas de información institucional.
5. Mayor impulso a un tipo de investigación y extensión universitaria vinculado a sus propias fortalezas, pero orientado a incidir en el desarrollo productivo, político, ambiental y social de las diferentes regiones del país.

6. Esfuerzos sistemáticos de promoción del desarrollo de capacidades profesionales del personal docente e investigativo universitario que promuevan y faciliten el acceso a posgrados y doctorados, capacitaciones específicas, participación en eventos significativos, congresos y pasantías.

En la UNED el PMI desarrollará nueve iniciativas, que estarán vinculadas a los cuatro pilares del FEES definidos por CONARE, como se muestra en la siguiente figura:

Iniciativas y su vinculación con los pilares definidos por CONARE
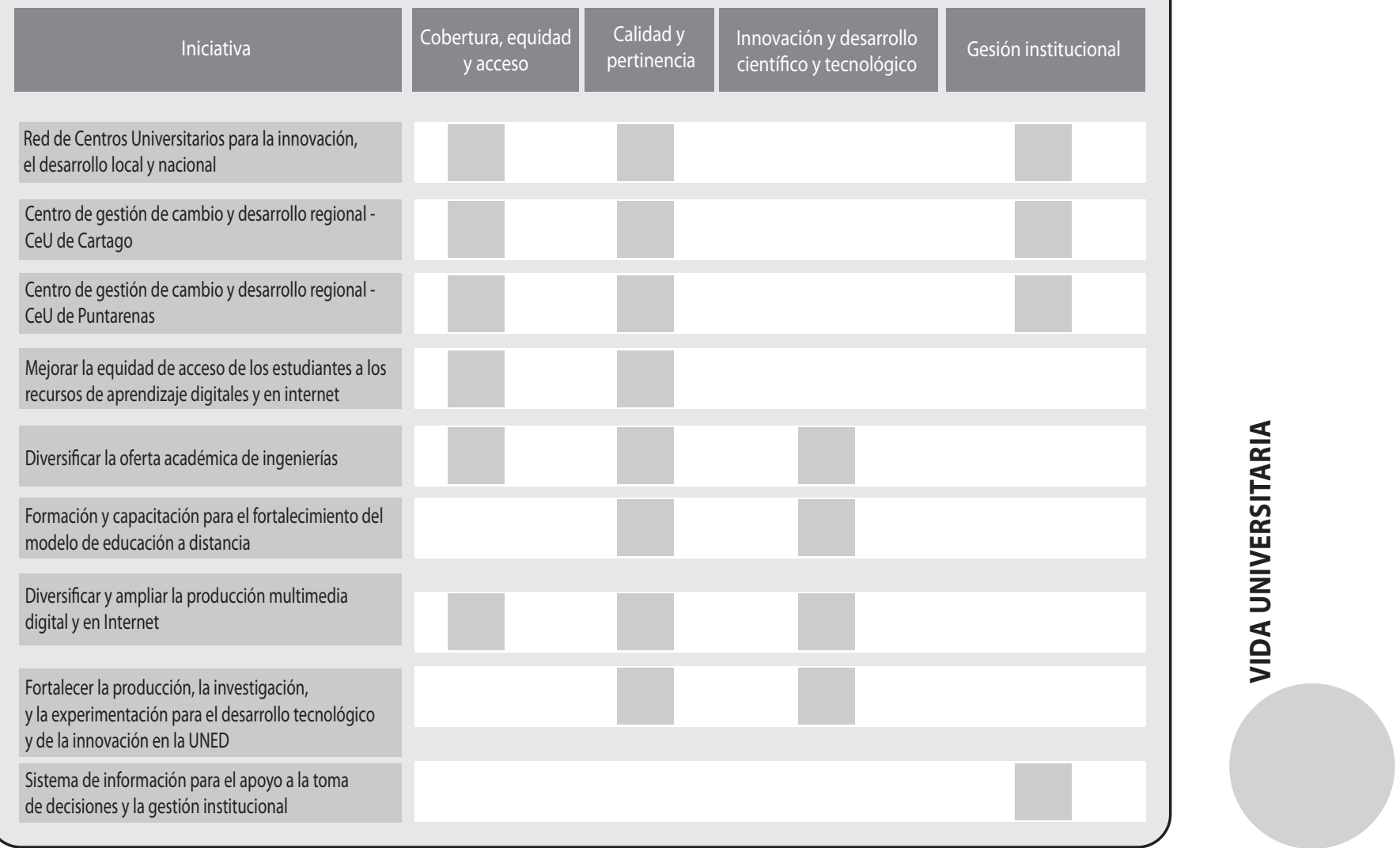


\section{Plan de mejoramiento institucional, iniciativas}

1. Red de Centros Universitarios para la innovación y el desarrollo local y nacional

2. Centro de Gestión de Cambio y Desarrollo Regional: Sede de Cartago

3. Centro de Gestión de Cambio y Desarrollo Regional: Sede de Puntarenas

4. Mejorar la equidad de acceso de los estudiantes a los recursos de aprendizaje digitales $\mathrm{y}$ en Internet

5. Diversificar la oferta académica de ingenierías

6. Formación y capacitación para el fortalecimiento del modelo de educación a distancia

7. Diversificar y ampliar la producción multimedia digital y en Internet

8. Fortalecer la producción, la investigación, la experimentación para el desarrollo tecnológico y de la innovación en la UNED

9. Sistema de información para el apoyo a la toma de decisiones y la gestión institucional

\section{Red de Centros Universitarios para la Innovación, el Desarrollo Local y Nacional}

Con esta iniciativa se pretende disminuir progresivamente la brecha educativa y de acceso, con Centros Universitarios que ofrezcan la misma calidad y cantidad de servicios en cualquier parte del territorio nacional. Se busca una solución de infraestructura integral y equitativa; que cubra las necesidades tecnológicas, de equipamiento e infraestructura. Complementando esta solución con la implementación de una conectividad más robusta a través de la instalación de fibra óptica o conexión de cobre de alta capacidad. Esto permitirá realizar una conexión segura, para brindar al estudiantado los servicios universitarios con eficiencia y eficacia; potenciar la innovación y la investigación en los procesos educativos y apoyar la virtualidad como complemento a la educación a distancia.
Centro de Gestión de Cambio y Desarrollo Regional: Centro Universitario de Cartago

Se construirá un Centro Universitario totalmente nuevo, la construcción abarcará $2198 \mathrm{~m}^{2}$. El centro contará con todos los servicios administrativos y académicos necesarios para satisfacer las demandas planteadas por el crecimiento de la matrícula, así como para mejorar el funcionamiento y las condiciones que permitirían la integración real de este centro en el desarrollo local.

\section{Centro de Gestión de Cambio y Desarrollo Regional: Centro Universitario de Puntarenas}

De igual manera que en Cartago, en Puntarenas se construirá un Centro Universitario totalmente nuevo, que tendrá un área de construcción de $2000 \mathrm{~m}^{2}$. El centro contará con todos los servicios administrativos y académicos necesarios para los estudiantes, con el fin de satisfacer las demandas planteadas por el crecimiento de la matrícula, así como para mejorar el funcionamiento y las condiciones para su integración real en el desarrollo local.

\section{Mejorar la Equidad de Acceso de los Estudiantes a los Recursos de Aprendizaje Digitales y en Internet}

Se pretende dotar de dispositivos móviles a un grupo de entre 1000 a 1500 estudiantes con limitaciones sociales y económicas, para garantizarles una inclusión real y equitativa en el uso de las TIC. Con esta iniciativa podrán tener acceso a los recursos didácticos de la UNED que se encuentran en línea (videos, audios, imágenes, multimedios, artículos y recursos educativos abiertos). La dotación de estos recursos tecnológicos a los estudiantes contribuiría a influir positivamente en el éxito académico, en la permanencia de los estudiantes en los programas de estudio y al término oportuno de los periodos de estudio de las personas beneficiadas. 


\section{Diversificar la Oferta Académica de las Ingenierías}

Se propone la apertura de tres ingenierías en la oferta académica de la UNED: Ingeniería Industrial, Ingeniería en Telecomunicaciones e Ingeniería Sanitaria. Todas bajo la modalidad de educación a distancia y con la intención de que abran el acceso a la educación en estas áreas, a aquellos sectores de la población que no han podido realizar sus estudios universitarios en opciones tradicionalmente presenciales y concentradas en el Gran Área Metropolitana.

\section{Formación y Capacitación para el Modelo de Educación a Distancia}

Se impulsará la formación de funcionarios, administrativos y académicos, mediante su participación en maestrías y doctorados, en procesos de capacitación y con la promoción de pasantías que fortalezcan el modelo de educación a distancia basado en las TIC y en disciplinas requeridas por la universidad.

\section{Diversificar y Ampliar la Producción Multimedia Digital y en Internet}

Para garantizar el acceso de los estudiantes a los recursos didácticos multimediales y audiovisuales, que contribuyen positivamente en los procesos de enseñanza y aprendizaje, se requiere de la actualización continua de los recursos técnicos para la producción digital multimedial.

\section{Fortalecer la Producción, la Investigación para el Desarrollo Tecnológico y la Innovación en la UNED}

Se prevé la construcción de un edificio, que se llamaría Ii+D, de aproximadamente $5945 \mathrm{~m}^{2}$ de construcción, que contará con la infraestructura, el equipamiento, los laboratorios especializados y las salas de reuniones con las condiciones y conectividad que se requiere. Además la iniciativa permitirá potenciar la adaptación y actualización de los procesos productivos y de los medios que se emplean para la creación y entrega de los recursos didácticos; el fortalecimiento de la investigación, la consolidación de la infraestructura tecnológica, elementos todos imprescindibles para, la innovación y el desarrollo en la UNED.

\section{Sistema de Información para el Apoyo a la Toma de Decisiones y a la Gestión Institucional}

Se prevé el fortalecimiento, mejoramiento y modernización de los sistemas de información institucionales, específicamente los que se relacionan con las áreas de recursos humanos, gestión académica, gestión de estudiantes y financierocontable. El propósito es integrarlos para que satisfagan de manera oportuna y pertinente las necesidades de información y de servicios que requieren los funcionarios del área estudiantil, académica y administrativa. Con este fortalecimiento se pretende, además, generar indicadores en áreas de interés institucional que apoyen la gestión y la toma de decisiones de las autoridades universitarias.

\section{Impacto del acuerdo de mejoramiento institucional en el modelo de educación a distancia}

\section{Red de centros universitarios para la innovación, el desarrollo local y nacional}

Como parte del fortalecimiento de la educación a distancia, el PMI establece una red de 37 centros universitarios que contarán con la misma cantidad y calidad de servicios, respondiendo siempre a las necesidades regionales y a la oferta de carreras en áreas definidas como relevantes.

Particularmente, los centros universitarios de Cartago y Puntarenas serán desarrolladas como puntos estratégicos de gestión de cambio y desarrollo local, ya que como otras, contarán con todos los servicios administrativos, recursos didácticos y tecnológicos necesarios para satisfacer las demandas planteadas por el crecimiento de la matrícula y para su integración real en el desarrollo local; apoyando una formación de calidad en nuestros estudiantes que les permita insertarse en el mercado laboral y aportar rápidamente al desarrollo de la economía 
de la región. Como resultado se espera una mejora sustancial de la condición de vida del estudiante, su familia y comunidad.

\section{Inversión en recursos didácticos digitales y tecnologías de la información y comunicación}

Para que nuestros estudiantes gocen de acceso a la tecnología necesaria para enriquecer significativamente sus procesos de enseñanza y de aprendizaje, la UNED hará una inversión importante en conectividad, en salas tecnológicas con acceso a video conferencia, laboratorios de cómputo, laboratorios de ingeniería, en el equipamiento de los laboratorios para la práctica docente, en la implementación de centros de recursos académicos para estudiantes y en la capacitación de los miembros de los centros universitarios. Aunado a este esfuerzo se contempla la ampliación, diversificación y acceso de los estudiantes a recursos didácticos multimedia, así como a dispositivos móviles y a bases de datos con publicaciones científicas y académicas y a muchos recursos creados por la comunidad mundial accesibles por medio de Internet.

Este desarrollo será apoyado por el fortalecimiento, mejora y modernización de los sistemas de información institucionales, con el fin de integrarlos para que satisfagan de manera oportuna y pertinente las necesidades de información y de servicios que se requieren en el área estudiantil, académica, administrativa, así como las necesidades de información de las autoridades universitarias para la toma de decisiones y gestión institucional.

\section{Diversificación de la oferta académica, formación y capacitación en el modelo de educación a distancia}

La oferta académica que se propone parte de una visión innovadora y flexible, bajo un modelo de educación a distancia que garantice la cobertura con equidad en todas las regiones del país, en especial las más alejadas del valle central. Por esa razón, la UNED diversifica la oferta académica fortaleciendo las actuales carreras e incursionando en nuevas ingenierías. Esta diversificación no sería posible, sin la inversión en la formación del cuerpo docente en maestrías y doctorados de alto nivel en sus áreas disciplinares y con la capacitación permanente en educación a distancia y las tecnologías de información y comunicación, que impulsen la investigación e innovación en todas las áreas estratégicas de la institución. 